\title{
Oscillation Modes in Free-Running Oscillators Loaded with Multi-Resonant Networks
}

\author{
Franco Ramírez, Almudena Suárez, Sergio Sancho \\ Communications Engineering Department, University of Cantabria, Santander, Spain
}

\begin{abstract}
An in-depth investigation of oscillation modes in free-running oscillators loaded with multi-resonance networks is presented. It focuses on the mechanisms leading to the coexistence of stable oscillation modes, which may give rise to uncertainty in the physical behaviour. The multiple periodic and quasi-periodic solutions are detected and related to the stability properties of the de solution and each of the periodic modes. Two different types of Hopf-bifurcation loci enable a global understanding of the circuit operation. The investigation is initially carried out with an analytical formulation and then extended to harmonic-balance simulations. The results have been experimentally validated through their application to a HEMT-based cross-coupled oscillator, at 0.65 and $2.4 \mathrm{GHz}$.
\end{abstract}

Index Terms - Oscillation modes, stability, bifurcation.

\section{INTRODUCTION}

A relevant problem in free-running oscillators with multiresonance loads, such as crystal oscillators, used as low phasenoise references [1], or dual-band oscillators [2-3], with application in multi-band communication systems, is the possible coexistence of stable oscillation modes within certain parameter regions. This gives rise to uncertainty in the circuit behaviour since either one or another mode may be observed depending on the initial conditions. Quasi-periodic solutions in which the two modes coexist concurrently are also possible and can be exploited in dual-band systems [4-5]. However, the complex and ill-understood dynamics of multi-mode oscillators may degrade their response and limit its potential applications, due to the lack of robustness and/or the onset of undesired regimes. This work presents a thorough bifurcation analysis of these circuits in the frequency domain, which should provide insight into the mechanisms for the generation and stabilization of its various operation modes. It focuses on non-concurrent periodic oscillation modes, while setting the theoretical basis for the quasi-periodic case.

Due to the complexity of the problem, the initial investigation will be based on a simplified model of the active device. This will enable an exhaustive analytical study, comprising four different aspects: stability analysis of the dc solution, calculation and stability analysis of the individual oscillation modes and bifurcation detection. Such comprehensiveness would be virtually impossible with numerical techniques. Once the instability phenomena have been identified, it will be possible to derive criteria ensuring that the periodic oscillation modes are stable in disjoint ranges of tuning parameter(s). The test-bench load network is chosen to illustrate the existence of these disjoint regions, which, as will be shown, are due to the intersection of the primary Hopf-bifurcation loci [6-8] of the individual oscillation modes. A demonstrator based on HEMTbased cross-coupled oscillator at 0.65 and $2.4 \mathrm{GHz}$ has been manufactured and measured.

\section{ANALYTICAL INVESTIGATION OF BIFURCATION BEHAVIOUR}

The active device and the multi-resonance network will be respectively modelled with the voltage-dependent current $i(v)=a v+b v^{3}$, where $a<0, b>0$, and the admittance function $Y_{L}(j \omega)$. The poles of the dc regime are the roots of the characteristic equation: $a+Y_{L}(\mathrm{~s})=0$. For illustration, the circuit in Fig. 1(a) has been considered. When reducing $a$ from $-0.02 \Omega^{-1}$ [Fig. 2(a)], each crossing of a pair of complexconjugate poles through the imaginary axis corresponds to a primary Hopf bifurcation $(\mathrm{H})$ [6]. There are three crossings: $\mathrm{H}_{\mathrm{p} 1}, \mathrm{H}_{\mathrm{p} 2}$ and $\mathrm{H}_{\mathrm{p} 3}$, at the respective frequencies $f_{1}=4 \mathrm{GHz}$, $f_{2}=0.65 \mathrm{GHz}$ and $f_{3}=1.3 \mathrm{GHz}$, which suggests the presence of three oscillation modes. To calculate the modes, the nonlinear current will be excited with a periodic voltage, which provides the describing function $Y(V)=a+3 / 4 b V^{2}$. The steady-state oscillation condition is:

$$
\begin{aligned}
& Y_{T}^{r}\left(V_{m}, \omega_{m}\right)=a+\frac{3}{4} b V_{m}^{2}+Y_{L}^{r}\left(j \omega_{m}\right)=0 \\
& Y_{T}^{i}\left(V_{m}\right)=Y_{L}^{i}\left(j \omega_{m}\right)=0
\end{aligned}
$$

where $m$ refers to the oscillation mode. In Fig. 2(b), the three periodic oscillation modes are represented versus $a$ (periodic curves $C_{1}, C_{2}$ and $C_{3}$ ) The $a$ values at which these curves are generated agree with $\mathrm{H}_{\mathrm{p} 1}, \mathrm{H}_{\mathrm{p} 2}$ and $\mathrm{H}_{\mathrm{p} 3}$. A Hopf bifurcation is supercritical if the generated curve exists only after the critical pair of complex-conjugate poles has crossed the imaginary axis to the right-hand side (RHP), as $\mathrm{H}_{\mathrm{p} 1}$ and $\mathrm{H}_{\mathrm{p} 2}$. If the periodic curve exists before the crossing of the critical poles to the RHP, it will be subcritical, as $\mathrm{H}_{\mathrm{p} 3}$. Let the dc solution have $k$ unstable poles before the pole crossing. According to the Central Manifold Theorem (CMT) [6], in the supercritical case, the newly generated solution will have $k$ unstable canonical poles (with frequencies comprised between 0 and $\omega_{m} / 2$ ). In the subcritical case, it will contain $k+1$ unstable poles. Thus, in the neighborhood of $\mathrm{H}_{\mathrm{p} 1}, \mathrm{H}_{\mathrm{p} 2}$ and $\mathrm{H}_{\mathrm{p} 3}$, the periodic solutions (in $C_{1}$, $C_{2}$ and $C_{3}$, respectively) should have 0,2 and 3 unstable poles. The unstable curves may become stable if under a further 
variation of the parameter the originally unstable poles cross to the left-hand side (LHP).

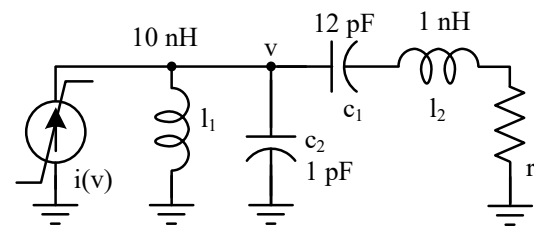

$\mathrm{i}(\mathrm{v})=-0.05 \mathrm{v}+0.5 \mathrm{v}$

(a)

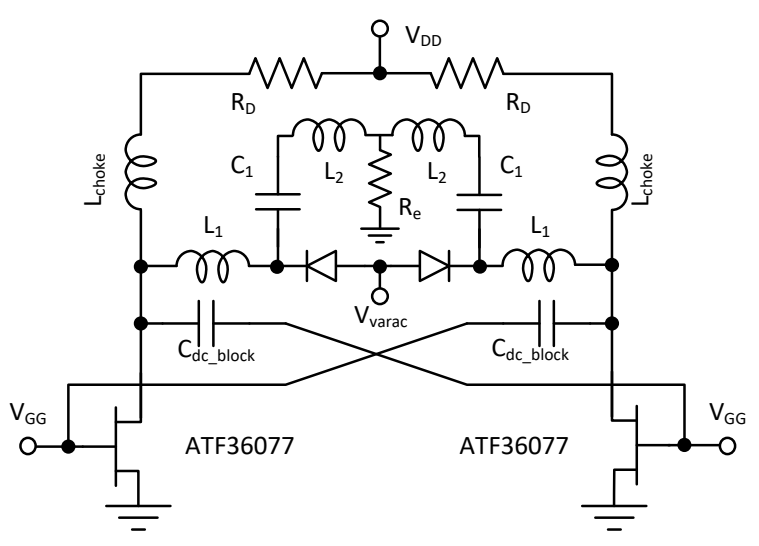

(b)

Fig. 1 (a) Simple circuit for the analytical study. (b) Experimental oscillator.

The stability analysis of each periodic oscillation mode will be carried out linearizing the active device about each periodic mode $m$, with the incremental describing function [9], $Y_{I}=a+3 / 2 V_{m}^{2}$, and making the passive network depend on the complex frequency s. This provides the following characteristic equation:

$$
a+3 / 2 b V_{m}^{2}+Y_{L}(s)=0
$$

This equation considers a real voltage $V_{m}$, so it intrinsically leaves out the pole at zero, associated with the oscillation autonomy. The resulting poles are shown in Fig. 2(c). The curve $C_{1}$ is always stable, whereas $C_{2}$ and $C_{3}$ are originally unstable. However, when further reducing $a$, the RHP poles in $C_{2}$ and $C_{3}$ cross to the LHP in different secondary Hopf bifurcations (from periodic regime) [6,7], at the point $\mathrm{H}_{\mathrm{s} 1}$ of $C_{2}$ and the points $\mathrm{H}_{\mathrm{s} 2}$ and $\mathrm{H}_{\mathrm{s} 3}$ of $C_{3}$ [Fig. 2(c)]. At a secondary Hopf bifurcation, a quasi-periodic solution arises from zero amplitude of the newly-generated oscillation component. These quasi-periodic solutions will be calculated exciting the nonlinear function $i(v)$ with the two-tone signal $V_{m} \cos \omega_{m} t+V_{n} \cos \omega_{n} t$, where $\omega_{m}$ and $\omega_{n}$ are incommensurate, and neglecting the intermodulation products [5]. A quasi-periodic solution at $\omega_{m}, \omega_{n}$ will fulfil:

$$
\begin{aligned}
& Y_{L}^{i}\left(j \omega_{m}\right)=0, \quad Y_{L}^{i}\left(j \omega_{n}\right)=0 \\
& Y_{1}^{r}\left(V_{m}, V_{n}, j \omega_{m}\right)=a+3 / 4 b V_{m}^{2}+3 / 2 b V_{n}^{2}+Y_{L}^{r}\left(j \omega_{m}\right)=0 \\
& Y_{2}^{r}\left(V_{m}, V_{n}, j \omega_{n}\right)=a+3 / 4 b V_{n}^{2}+3 / 2 b V_{m}^{2}+Y_{L}^{r}\left(j \omega_{n}\right)=0
\end{aligned}
$$

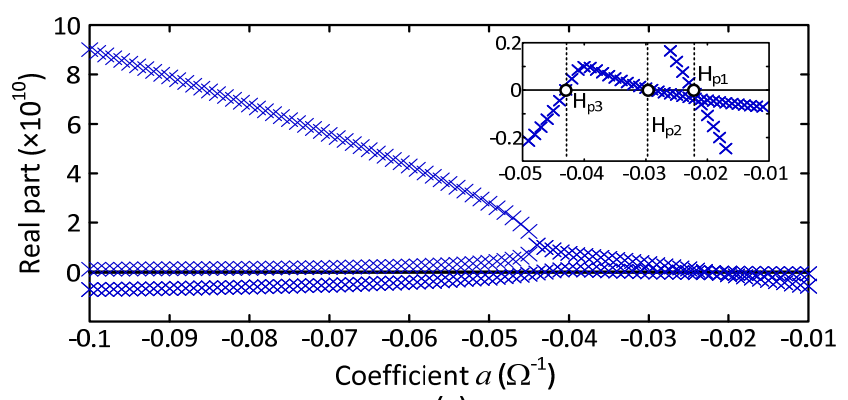

(a)

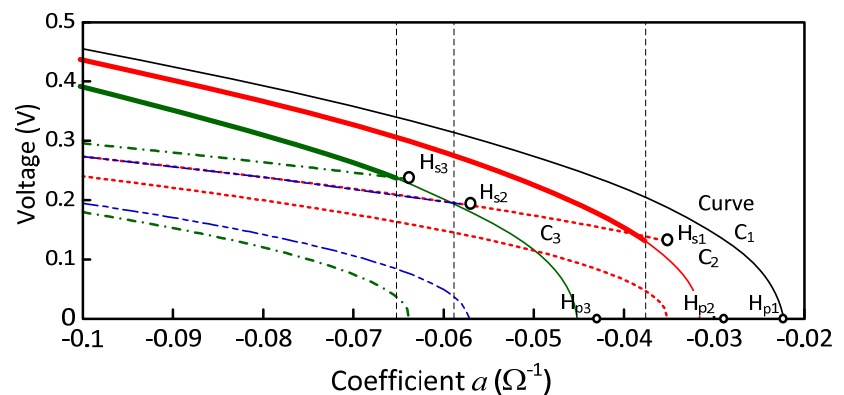

(b)

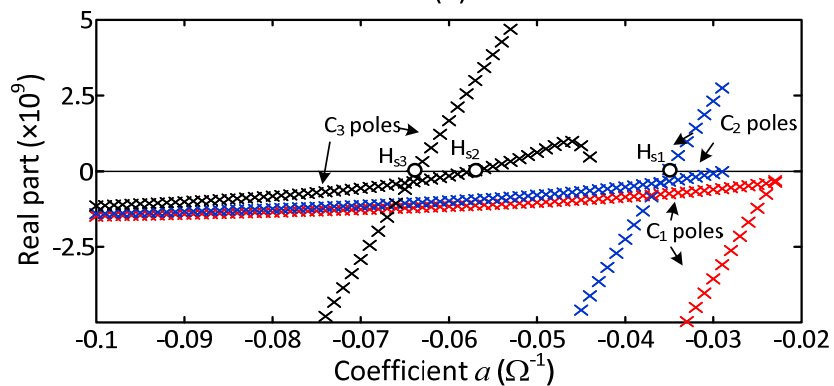

(c)

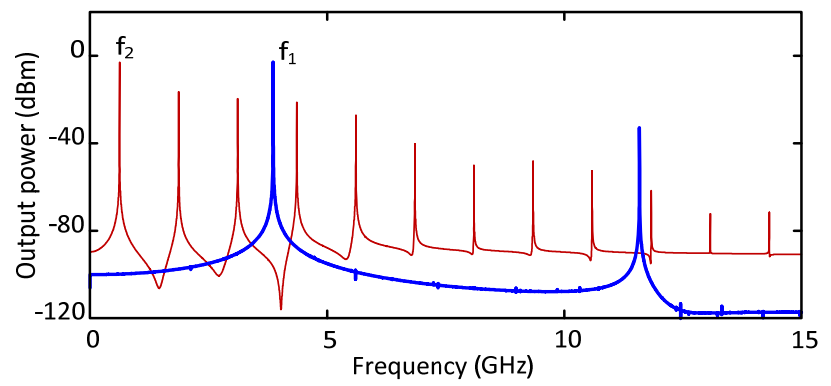

(d)

Fig. 2 Analytical investigation. (a) Poles of the dc solution. (b) Periodic and quasi-periodic solution curves, the latter generated at $\mathrm{H}_{\mathrm{s} 1}, \mathrm{H}_{\mathrm{s} 2}, \mathrm{H}_{\mathrm{s} 3}$. (c) Poles of the periodic solutions. (d) Validation of the coexistence of modes 1 and 2 with an independent transient analysis.

Three modes have been detected, so there are three possible combinations of $\omega_{m}, \omega_{n}$ values. Once $\omega_{m}$ and $\omega_{n}$ have been obtained from (a), subsystem (b) and (c) can be analytically solved in $V_{m}^{2}$ and $V_{n}^{2}$. The quasi-periodic solutions have been included in Fig. 2(b) and their onset (from zero value of the newly generated oscillation components) agrees with the predictions of the pole analysis in Fig. 2(c). At a secondary Hopf bifurcation the stability properties of the bifurcating periodic solution are transferred to the generated quasi-periodic 
one, following a rule analogous to the one in primary Hopf bifurcations [6]. The three bifurcations $\mathrm{H}_{\mathrm{s} 1}, \mathrm{H}_{\mathrm{s} 2}$ and $\mathrm{H}_{\mathrm{s} 3}$ are subcritical and give rise to unstable quasi-periodic solutions (in agreement with [5]), with 1, 3 and 1 unstable canonical poles, respectively. Despite their instability, these solutions have a fundamental impact on the circuit behaviour. For $a<a\left(\mathrm{H}_{\mathrm{s} 1}\right)$, the two periodic oscillations at $f_{1}=4 \mathrm{GHz}$ and $f_{2}=0.65 \mathrm{GHz}$, are stable. Both may be physically observed, depending on the initial values, as verified with independent transient analyses in a commercial simulator [Fig. 2(d)], for $a=-0.04 \Omega^{-1}$. Good agreement is found despite the one-harmonic approximation in the analytical study.

For a global comprehension of the stability properties, the primary and secondary Hopf bifurcation loci will be calculated versus $a$ and the capacitor $c_{1}$. The primary Hopf loci are obtained by setting $V_{m}$ to zero in the equations (1) and solving for $a$ and $c_{1}$. The Hopf locus associated with a periodic solution at $\omega_{m}$, from which an oscillation at $\omega_{n}$ is generated, is obtained by sweeping $c_{1}$ and solving the following system, for $a$ and $V_{m}$, at each step:

$$
\begin{aligned}
& Y_{L}^{i}\left(j \omega_{m}\right)=0, \quad Y_{L}^{r}\left(j \omega_{n}\right)=0 \\
& Y_{T}^{r}\left(V_{m}, V_{n}=0, j \omega_{m}\right)=3 / 4 b V_{m}^{2}+a+Y_{L}^{r}\left(j \omega_{m}\right)=0 \\
& Y_{T}^{r}\left(V_{m}, V_{n}=0, j \omega_{n}\right)=3 / 2 b V_{m}^{2}+a+Y_{L}^{r}\left(j \omega_{n}\right)=0
\end{aligned}
$$

The Hopf loci (Fig. 3) divide the plane in regions with different qualitative behavior. At the self-intersection point of the primary Hopf locus $\left(X_{1}\right)$, there are two pairs of poles $\pm j 2 \pi f_{1}$ and $\pm j 2 \pi f_{2}$ on the imaginary axis. Above (below) this point, the oscillation at $f_{1}\left(f_{2}\right)$ is generated when crossing this locus from the non-oscillation region. In each case, this will be the only stable solution until reaching the secondary Hopf locus (see arrows at $f_{1}$ and $f_{2}$ ). The primary Hopf loci must intersect to ensure regions with a single stable periodic mode. The boundary between the regions with a single stable periodic solution at $f_{1}$ (or $f_{2}$ ) and two stable periodic solutions at $f_{1}$ and $f_{2}$ have been calculated with an independent transient analysis, with the results superimposed with asterisks.

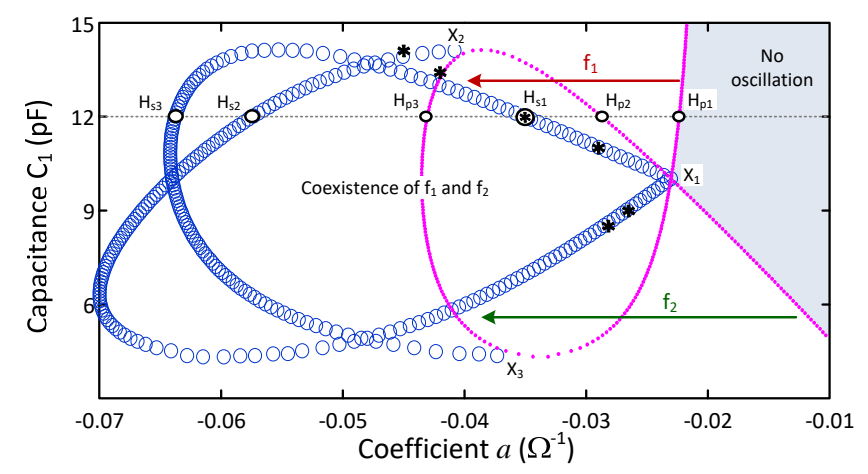

Fig. 3 Bifurcation analysis. Regions in which the periodic modes at $f_{1}$ and $f_{2}$ exist as single stable solutions are bounded by the primary (dots) and secondary (circles) Hopf loci. Results from transient simulations are superimposed with asterisks $(*)$.

\section{APPLICATION TO A CROSS-COUPLED OSCILLATOR}

A dual-frequency cross-coupled oscillator [Fig, 1(b)] has been designed as a demonstrator. The tuning parameters are the varactor capacitance and the drain voltage $V_{D D}$, although a different parameter could be used in a more advanced design. The gate voltage has been purposely avoided due to the structural occurrence of hysteresis. Two odd-mode resonances are obtained, at $f_{1}=0.65 \mathrm{GHz}$ and $f_{2}=2.4 \mathrm{GHz}$. The primary Hopf loci have been traced by connecting a small signal current source between the drain nodes of the two transistors and imposing a zero value of the total input admittance, which constitutes a limit oscillation condition [7]: $Y_{s s}\left(V_{D D}, c_{1}, \omega_{\mathrm{m}}\right)=0$, where $m=1,2$ refers to the oscillation mode. The two primary Hopf bifurcation loci have been traced in Fig. 4, with measurement points superimposed.

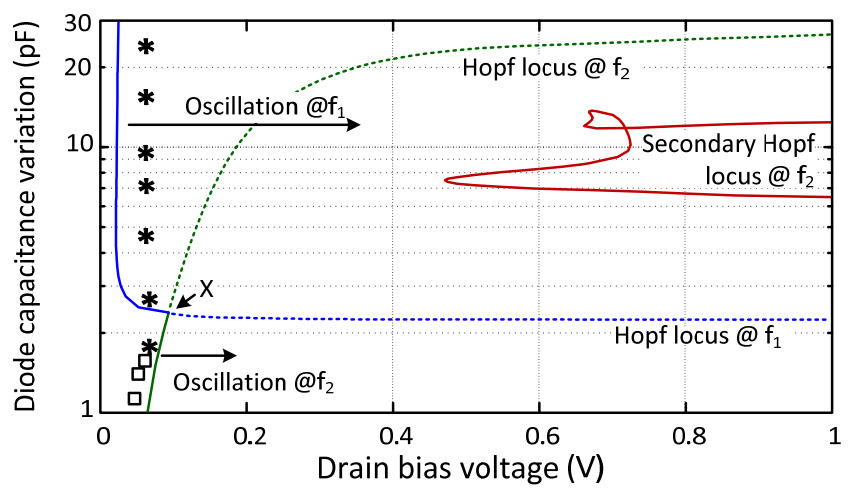

Fig. 4 Hopf loci of the oscillator in Fig. 1(b), dividing the plane into regions with different qualitative behavior. Measurements have been superimposed.

For very low $V_{D D}$, there is no oscillation, as in the case of Fig. 3 for big $a$ values. When increasing $V_{D D}$ the circuit oscillates at $f_{1}\left(f_{2}\right)$ above (below) the point $X$. Above $X$, there is initially only one stable solution at $f_{1}$. When crossing the primary Hopf locus at $f_{2}$, a second mode arises at $f_{2}$. It is initially unstable but becomes stable when crossing the secondary Hopf bifurcation locus. This locus has been calculated introducing into the circuit an auxiliary generator at $\omega_{A G}=\omega_{2}=2 \pi f_{2}$ [7] to obtain the steady-state oscillation, with 7 harmonic terms, and a smallsignal current source at an incommensurate frequency $f$, both connected between the drain nodes of the two transistors. The secondary Hopf bifurcation locus is obtained by solving the outer-tier system:

$$
\begin{aligned}
& Y_{A G}\left(A_{A G}, \omega_{2}, V_{D D}, c_{1}\right)=0 \\
& Y_{s S}\left(A_{A G}, \omega_{2}, V_{D D}, c_{1}, f\right)=0
\end{aligned}
$$

where (a) is a HB equation and (b) is a conversion-matrix equation. The loci divide the parameter plane into a region without oscillation, two regions with single stable oscillations and a more complex region (inside the secondary Hopf locus) where two stable oscillations coexist and even give rise to stable quasi-periodic modes. Within the single-stability regions, a robust experimental behavior was obtained (Fig. 5), even under 
significant changes of the initial conditions. No special care has been taken to reduce the harmonic content in this proof-ofconcept oscillator. Moreover, it is well-suited for a future analysis of the synchronization of the oscillations [6], their stability [10], phase noise [11] and other possible applications [12-14].

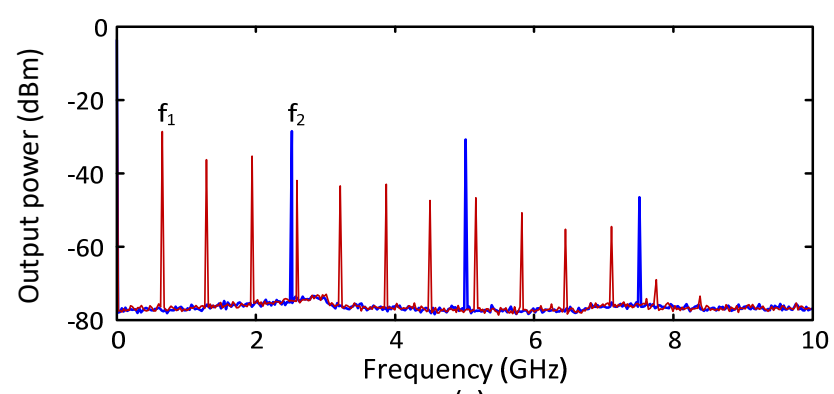

(a)

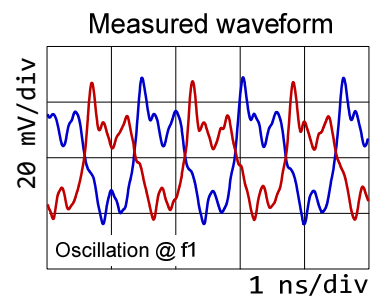

(b)

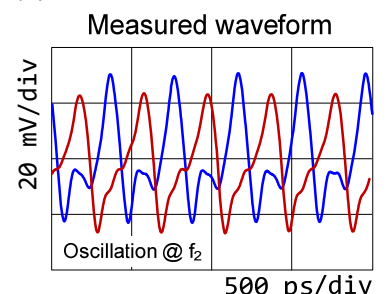

(c)
Fig. 5 Measurements of the HEMT based oscillator using a microstrip coupledline coupler. (a) Spectra of the two modes at 0.65 and $2.4 \mathrm{GHz}$. (b) $0.65 \mathrm{GHz}$ : $\mathrm{V}_{\mathrm{DD}}=0.29 \mathrm{~V}, \mathrm{~V}_{\mathrm{GG}}=-0.6 \mathrm{~V}$. (c) $2.4 \mathrm{GHz}: \mathrm{V}_{\mathrm{DD}}=0.22 \mathrm{~V}, \mathrm{~V}_{\mathrm{GG}}=-0.6 \mathrm{~V}$.

\section{CONCLUSION}

A detailed theoretical study of the behavior of multi-mode oscillators has been presented, evidencing the mechanisms for an undesired coexistence of oscillation modes. A criterion to isolate the modes in disjoint regions of the turning-parameter plane has been derived and experimentally demonstrated with a cross-coupled oscillator at 0.65 and $2.4 \mathrm{GHz}$.

\section{ACKNOWLEDGEMENT}

This work has been supported by the Spanish Ministry of Economy and Competitiveness under project TEC2014-60283C3-1-R and the Parliament of Cantabria (12.JP02.64069). The authors would like to thank S. Pana, University of Cantabria, for her assistance with the manufacturing process.

\section{REFERENCES}

[1] A. Ballato, "Piezoelectric Resonators," in B. Parzen, Design of Crystal and Other Harmonic Oscillators, pp. 66-122, John Wiley and Sons, 1983.

[2] Z. Chengjie, et al., "Dual-Mode Resonator and Switchless Reconfigurable Oscillator Based on Piezoelectric AlN MEMS Technology," IEEE Trans. on Electron Devices, vol.58, no.10, pp .35993603, Oct. 2011.

[3] U. Rohde, A. Poddar, "Concurrent, reconfigurable and adaptable oscillators for multiband multimode communication systems," 37th European Microwave Conference, 2007, pp. 1050-1053.
[4] A. Goel and H. Hashemi, "Injection Locking in Concurrent DualFrequency Oscillators," IEEE Trans. Microw. Theory Techn., vol.56, no.8, pp.1834-1845, 2008.

[5] J. Guckenheimer and P. Holmes, Nonlinear Oscillations, Dynamic Systems, and Bifurcations of Vector Fields, Springer-Verlag, 1983.

[6] J. S. Schaffner, "Simultaneous oscillations in oscillators", Transactions of the IRE, vol.CT-1, no.2, pp.2-8, June 1954.

[7] A. Suárez, Analysis and Design of Autonomous Microwave Circuits. Hoboken, New Jersey: John Wiley \& Sons, 2009.

[8] A. Suárez, R. Quéré, Stability Analysis of Nonlinear Microwave Circuits, Artech-House Publishers, Boston, 2003.

[9] A. Gelb, W. Vander Velde, Multiple-input describing functions and nonlinear system design, McGraw-Hill, 1968.

[10] S. Sancho, F. Ramírez and A. Suárez, "General stabilization techniques for microwave oscillators," IEEE Microw. and Wireless Comp. Lett., vol. 15, pp. 868-870, Dec., 2005.

[11] A. Suárez and F. Ramírez, "Analysis of stabilization circuits for phasenoise reduction in microwave oscillators," IEEE Trans. Microwave Theory Tech., vol. 53, pp. 2743-2751, Sept., 2005.

[12] M. Pontón, E. Fernández, A. Suárez, F. Ramírez, "Optimized design of pulsed waveform oscillators and frequency dividers," IEEE Trans. Microw. Theory Techn., vol. 59, no. 12, pp. 3428-3440, Dec., 2011.

[13] F. Ramírez, M. Pontón, S. Sancho, A. Suárez, "Stability analysis of oscillation modes in quadruple-push and Rucker's oscillators," IEEE Trans. Microw. Theory Techn., vol. 56, no. 11, pp. 2648-2661, Nov., 2008.

[14] E. de Cos, A. Suárez and S. Sancho, "Envelope transient analysis of selfoscillating mixers," IEEE Trans. Microwave Theory Tech., vol. 52, pp. 1090-1100, 2004. 